\title{
Ipsilateral simultaneous inferior shoulder dislocation and posterior elbow dislocation: A case report
}

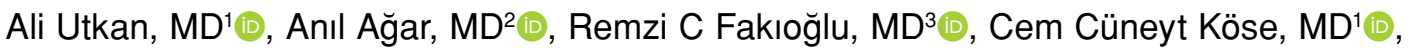 \\ Bülent Özkurt, MD' ${ }^{1}$
}

1'Department of Orthopedics and Traumatology, University of Health Science Ankara Bilkent City Hospital, Ankara, Turkey 2Department of Orthopedics and Traumatology, Istanbul Kanuni Sultan Süleyman Training and Research Hospital, Istanbul, Turkey ${ }^{3}$ Department of Orthopedics and Traumatology, Hitit University Erol Olçok Training and Research Hospital, Çorum, Turkey

Although the shoulder joint is the most frequently dislocated joint followed by the elbow joint, simultaneous shoulder and elbow dislocation on the same limb seldom occurs and there are only few cases in the literature. ${ }^{[1-8]}$ Moreover, inferior glenohumeral dislocation (luxatio erecta humeri) is rarely seen, accounting for less than $1 \%$ of all shoulder dislocations. The following unique patient had ipsilateral simultaneous inferior shoulder dislocation and posterior elbow dislocation. Such a combination of injury possibly occurs; however, to the best of our knowledge, this is the first reported case.

\section{CASE REPORT}

A 21-year-old male construction worker was brought to the emergency department in an ambulance after

Received: March 19, 2020

Accepted: June 20, 2020

Published online: September 11, 2020

Correspondence: Ali Utkan, MD. 1489. Cadde, Kardelen Sitesi, C Blok, No: 7/20 Yüzüncüyıl İşciblokları Mahallesi, 06530 Çankaya, Ankara, Türkiye.

E-mail: utkana@yahoo.com

Doi: $10.5606 /$ ehc. 2020.74939

Citation: Utkan A, Ağar A, Fakıoğlu RC, Köse CC, Özkurt B. Ipsilateral simultaneous inferior shoulder dislocation and posterior elbow dislocation: A case report. Jt Dis Relat Surg 2020;31(3):614-618

(C)2020 All right reserved by the Turkish Joint Diseases Foundation

This is an open access article under the terms of the Creative Commons Attribution-NonCommercial License, which permits use, distribution and reproduction in any medium, provided the original work is properly cited and is not used for commercial purposes (http://creativecommons.org/licenses/by-nc/4.0/).

\section{ABSTRACT}

This case report presents a 21 -year-old male construction worker with ipsilateral inferior shoulder and posterior elbow dislocations, accompanied with other injuries. Such a combination of injury undoubtedly occurs; however, to the best of our knowledge, this is the first reported case. The prompt closed reductions without waiting for anesthesia were accomplished without the help of an assistant using a novel technique. The patient returned to his job with considerable recovery of motion and strength despite his permanent paralysis of the deltoid muscle and he was still employed as a worker after four years. He was abducting his arm by using his accessory muscles, which contribute to abduction when arm is externally rotated. The maneuver described in the report can be used to reduce all inferior shoulder dislocations and it is worth to know that working at a job requiring high level of activity is still possible despite a permanent loss of axillary nerve function.

Keywords: Elbow dislocation, inferior shoulder dislocation, luxatio erecta humeri, shoulder dislocation, simultaneous dislocation.

falling from an eight-meter high platform. He was on a spine board and a cervical collar was applied. He was conscious and cooperative but was in great pain. He was not able to define what had happened and how he fell but probably he attempted to grip the scaffolding during his fall. He was holding his left arm above his head in a hyperabducted position and was unable to change his posture. Attempts to bring his arm down towards his trunk were failed due to mechanical resistance and significant pain. Also, his elbow was deformed and he was keeping it in flexed position. His left radial pulse was barely palpable. A quick neurologic examination was performed but unfortunately the axillary nerve was not assessed at that time. There were open skin wounds on the right foot, right calf and left hand. A written informed consent was obtained from the patient. 

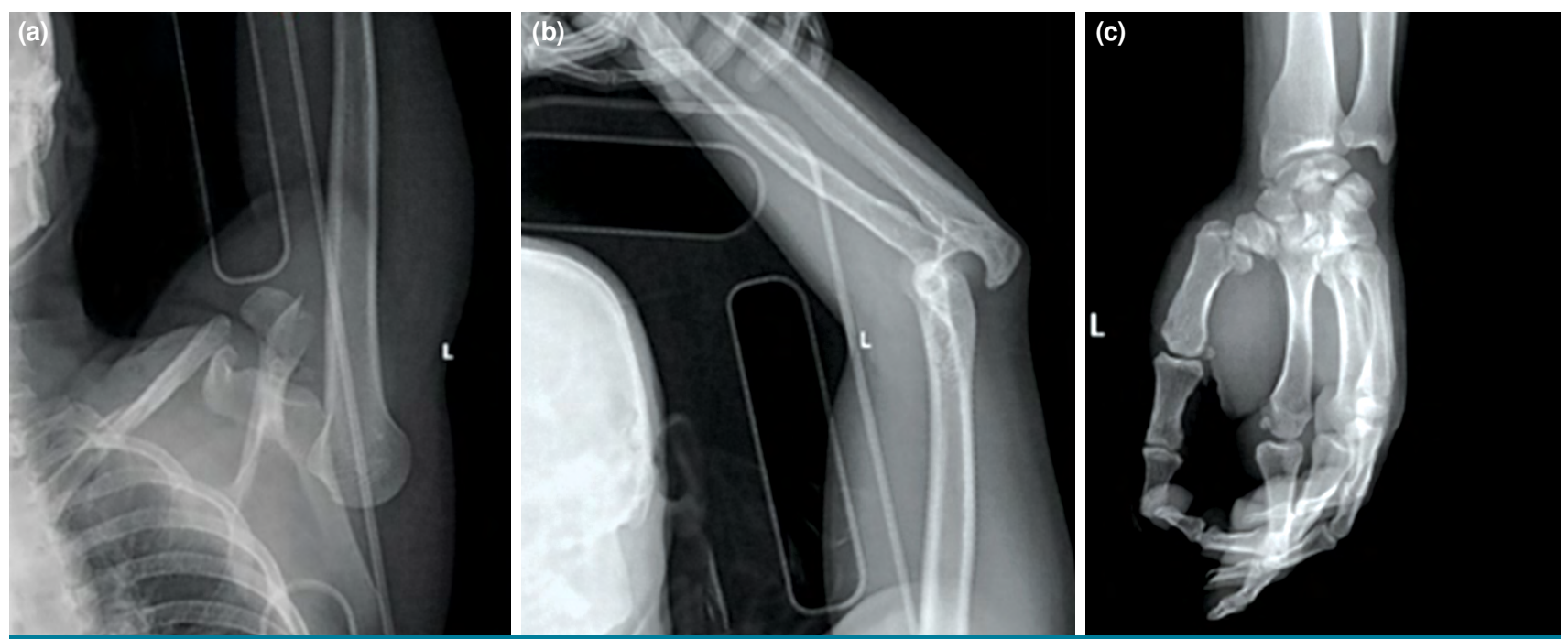

FIGURE 1. Initial X-rays of left shoulder, elbow and hand.

(a) Inferior shoulder dislocation and clavicle fracture.

(b) Posterior dislocation of elbow. (c) Scaphoid and Bennett fractures.

Radiological evaluation revealed the diagnosis of inferior shoulder dislocation and posterior elbow dislocation (Figure 1). There were also fractures of clavicle, scaphoid, base of the first metacarpal extending into the carpometacarpal joint (Bennett fracture) all in the same side (left) and calcaneus fracture at the contralateral side. The emergency physicians ordered a whole body computed tomography (CT) as it is considered a routine workup for multi-trauma patients to detect possible soft tissue injuries of the chest and abdomen. Considering the situation, the senior surgeon (first author) decided to reduce elbow and convert the inferior shoulder dislocation into an anterior dislocation straightaway while patient was still on the X-ray table without administering any medication as it would have had caused delay. Standing at the head of the table, he distracted the patient's flexed elbow to yield translation and felt the reduction and tested it by elbow passive range of motion. The surgeon flexed his own left elbow to 90 degrees and adducted his arm firmly, then, grasped patient's left elbow and wrapped it by his fingers so that the patient's elbow was kept in slightly flexed position. He placed his right hand's palm on the patient's chest to provide counter traction so that volar side of surgeon's wrist was lightly touching and leveraging the dislocated humeral head (Figure 2a). Then, he twisted his torso in counterclockwise direction to generate upward and outward traction (Figure $2 b$ ) and gradually adducted the patient's arm by taking small side steps, changing his position but maintaining the traction generated by his dynamic trunk posture (Figure 2c, d). Finally, patient's arm was adducted against the body. (a)

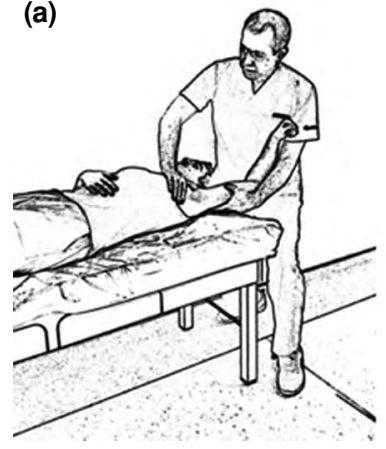

(b)

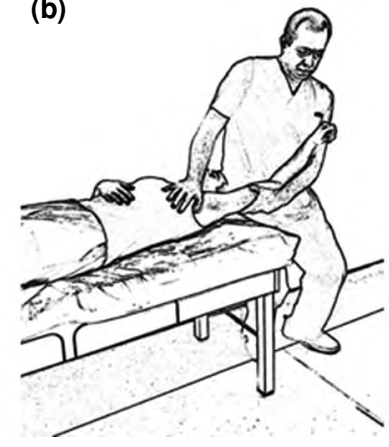

(c)

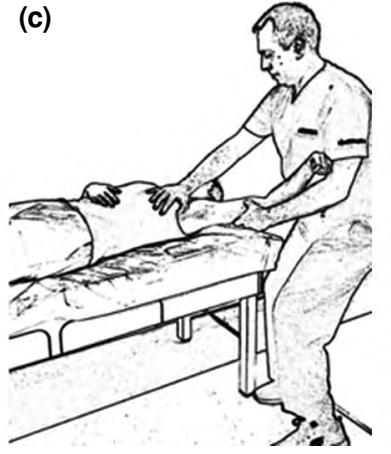

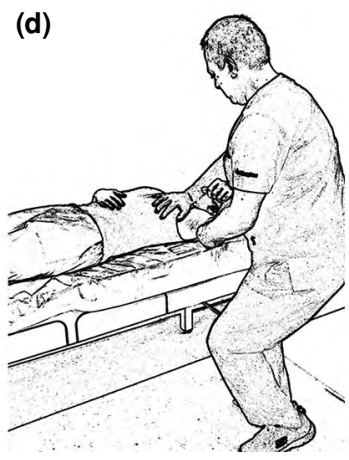

FIGURE 2. Reduction maneuver with schematic figures. 


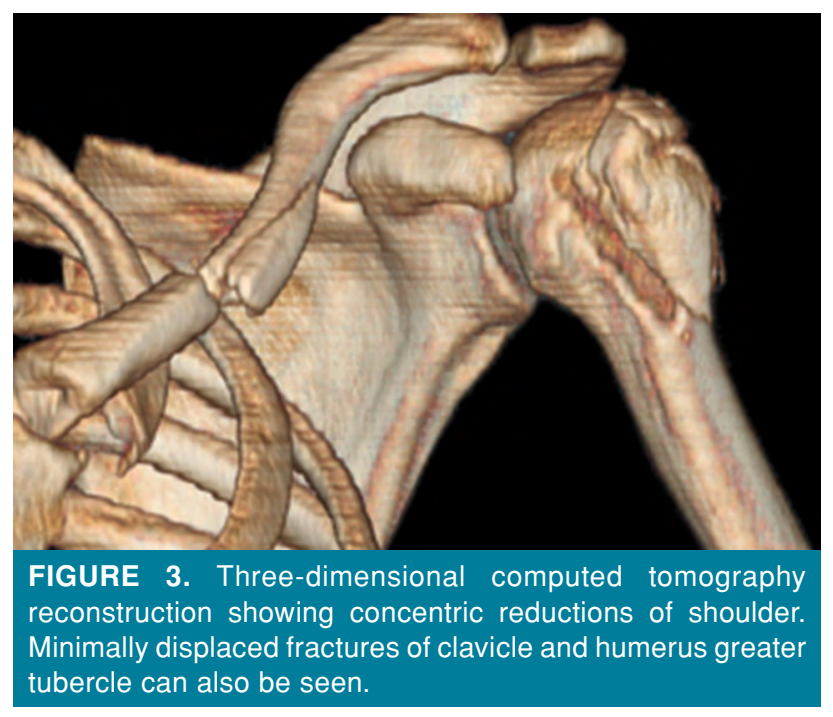

Surprisingly, the patient responded with a complete relief of pain. Radial pulse was checked and found normal. X-rays obtained subsequently showed that both dislocations were reduced. Then, a long arm plaster splint and a Velpeau bandage were applied. Afterwards, further evaluation and treatment of the patient proceeded.

Shoulder and elbow joints were also included in the total body CT scan. Both joints were reduced

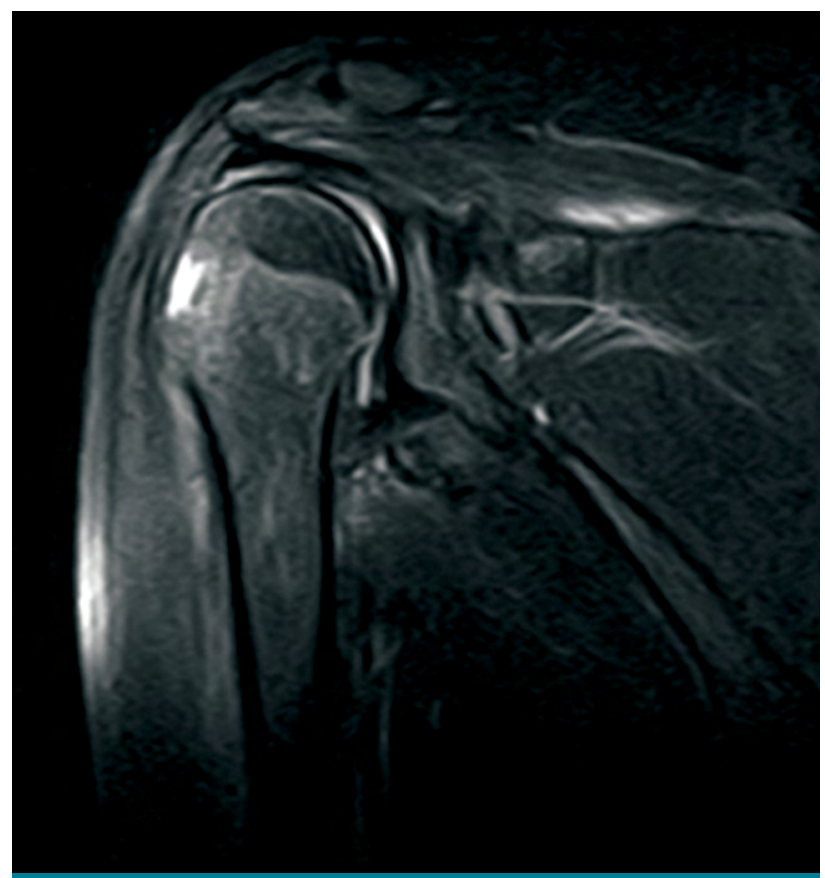

FIGURE 4. Coronal T1-weighted magnetic resonance image of shoulder demonstrating complete rupture of supraspinatus tendon. Loss of deltoid muscle mass is obvious. but there was a minimally displaced humerus greater tubercle fracture (Figure 3). Fortunately, no additional serious injury that necessitated emergent surgical intervention was detected and cleaning and suturing of wounds were accomplished under local anesthesia. A posterior short leg plaster splint was applied to right side. Next day, scaphoid fracture was fixed with headless compression screw and Bennett fracture with K-wires percutaneously under regional anesthesia. The elbow was reexamined and found stable. The clavicle and calcaneus fractures were managed conservatively. After completion of review diagnostic studies and multidisciplinary care, the patient was discharged at sixth day.

The patient was followed-up regularly and appropriately. The Velpeau bandage immobilization was discontinued and K-wires were removed at fifth week. Pendulum exercises for shoulder and passive range of motion exercises for elbow were initiated. At sixth week, a rotator cuff strengthening program was initiated. At third month, the range of motion at left shoulder was not satisfactory and magnetic resonance imaging was performed which revealed complete rupture of the supraspinatus tendon (Figure 4).

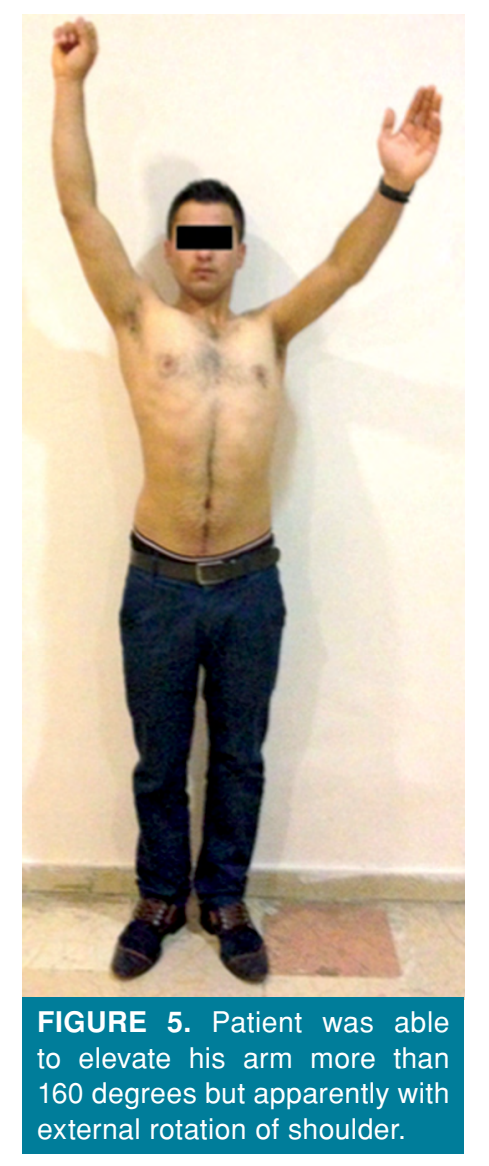


Loss of deltoid muscle mass was also noticeable. Then, rotator cuff repair with mini open exposure was performed. Six months after the injury, there was obvious wasting of the deltoid muscle and left brachial plexus electromyography revealed isolated axillary nerve lesion. However, the patient was elevating the left arm approximately 160 degrees without any pain apparently with compensatory action of accessory muscles and external rotation of shoulder as well as by increased rotation of the scapula (Figure 5). The patient was referred to a hand surgeon and no additional intervention was recommended for axillary nerve lesion. All fractures healed clinically and radiographically and the patient returned to his work as a construction worker with considerable recovery of motion and strength at ninth month. Four years after the injury, he was still employed as a worker.

\section{DISCUSSION}

Simultaneous presentation of shoulder and elbow dislocation on the same side is an extraordinary injury and only few cases have been reported in the literature. ${ }^{[1-8]}$ In some of them, the shoulder joint dislocation was initially missed and subsequently detected in the follow-up. ${ }^{[1,4,7]}$ The concomitance of inferior glenohumeral dislocation in this multiply injured patient makes the case unique, and to the best of our knowledge, this is the first reported case. ${ }^{[9]}$

Having the most extensive range of motion of any joint in the human body, the shoulder dislocation is the most common large joint dislocation seen in the emergency department. ${ }^{[10]}$ The majority of dislocations are anterior where less than $3 \%$ are posterior. ${ }^{[11]}$ Inferior glenohumeral dislocation is rare, accounting for less than $1 \%$ of all shoulder dislocations. ${ }^{[12]}$ On the other hand, the elbow is an inherently stable joint owed to congruous joint surfaces and surrounding capsuloligamentous structures, hence the elbow dislocation is relatively rare. Over $90 \%$ of elbow dislocations occur in a posterior or posterolateral direction. Typically, simple elbow dislocation is treated by closed reduction by gentle manipulation. Taylor et al. ${ }^{[13]}$ reviewed various randomized controlled trials on the treatment of simple dislocations of the elbow in adults to determine the best type and duration of immobilization after reduction but concluded that there is insufficient evidence to suggest the most appropriate method of treatment. In the presented case, the reduction of the elbow was undoubtedly easy and the elbow was immobilized for five weeks due to accompanying fractures.

Inferior dislocations are particularly unique, as traditional anterior and posterior dislocation reduction techniques are not appropriate for this type of dislocation. The most effective reported reduction technique is the traction-countertraction technique. ${ }^{[14]}$ Upward traction on the extended arm is applied in line with the humeral shaft while an assistant applies countertraction in the opposite direction. The other technique described by Nho et al. ${ }^{[15]}$ is called the two-step maneuver where the inferior dislocation is converted into an anterior dislocation and then reduced by one of the known methods.

Although delays in reducing dislocated joints could be accepted in life-threatening conditions, the bizarre posture of the patient necessitated immediate intervention before further management. Although only converting inferior dislocation into an anterior dislocation was planned via the aforementioned technique, the shoulder was reduced at first attempt. Using torso and changing position of body instead of pushing or pulling by arm to produce stronger force is a known technical gimmick in aikido and, in this situation, helped the surgeon to accomplish reduction without the need of an assistant in this situation.

Groh et al. ${ }^{[16]}$ reported the largest series of patients, which included 20 shoulders in 18 patients treated for inferior shoulder dislocation at a single institution. All patients were initially managed with closed reduction, which was successful in nine shoulders. The remaining nine shoulders required operative treatment. Mallon et al. ${ }^{[17]}$ reviewed the literature and found 80 cases. In these two reports, rotator cuff tear or fracture of the greater tuberosity was found in $80 \%$ of the patients while associated injuries were listed as peripheral nerve injury, humeral fracture, acromial fracture and rotator cuff tear. Although there are some reports in which patients functionally recovered by conservative treatment, some authors preferred immediate reduction followed by surgical repair of the rotator cuff at a later date as we did.

The humerus greater tubercle fracture was undisplaced and internal fixation was not considered in this patient. Schliemann et al. ${ }^{[18]}$ analyzed the results of 105 conservatively treated patients having an isolated fracture of the greater tuberosity and concluded that surgery is most likely required for associated soft tissue lesions rather than for secondary displacements. The fracture healed without secondary displacement in this patient in five weeks.

One of the problems that can be criticized in the management of this case is that a detailed 
examination of the axillary nerve was missed both before and after the reduction. Isolated axillary nerve lesion was detected during follow-up. The literature provides conflicting viewpoints on surgical treatment of axillary nerve injuries. ${ }^{[19]}$ Nevertheless, after permanent loss of axillary nerve function, working at a job requiring high level of activity is possible. ${ }^{[20]}$ Palmer and Ross ${ }^{[21]}$ presented three cases of proven complete paralysis of the deltoid with an almost full range of movement of the shoulder owing to the compensatory action of accessory muscles. The presented patient was also abducting his arm by using accessory muscles, which contribute to abduction when shoulder is externally rotated. The abductor moment arm of the subscapularis muscle generally increases with external rotation and the long head of the biceps muscle assists with abduction when the shoulder is externally rotated. In this way, the patient was able to return to his employment with considerable recovery of motion and strength.

In conclusion, we have reported a unique case with ipsilateral inferior shoulder and posterior elbow dislocation with accompanying fractures. The reduction technique described in the report is quick, simple and requires neither an assistant nor anesthesia and thus can be used in all inferior shoulder dislocations. The denervation of the deltoid muscle that is one of the complications of shoulder dislocations needs not be associated with significant disability in every patient and restoration of function with minimal loss can be expected with good rehabilitation.

\section{Declaration of conflicting interests}

The authors declared no conflicts of interest with respect to the authorship and/or publication of this article.

\section{Funding}

The authors received no financial support for the research and/or authorship of this article.

\section{REFERENCES}

1. Ali FM, Krishnan S, Farhan MJ. A case of ipsilateral shoulder and elbow dislocation: an easily missed injury. J Accid Emerg Med 1998;15:198.

2. Cobanoğlu M, Yumrukcal F, Karataş C, Duygun F. Simultaneous shoulder and elbow dislocation. BMJ Case Rep 2014;2014:bcr2014204686.

3. Sié Essoh JB, Kodo M, Traoré A, Lambin Y. Ipsilateral dislocation of the shoulder and elbow: A case report. Niger J Surg Res 2005;7:319-20.
4. İmerci A, Kumbaracı K, İncesu M, Savran A, Karapınar L. Ipsilateral simultaneous shoulder and elbow dislocation: A case report. Tr J Emerg Med 2011;11:72-5.

5. Inan U, Cevik AA, Omeroğlu H. Open humerus shaft fracture with ipsilateral anterior shoulder fracturedislocation and posterior elbow dislocation: a case report. J Trauma 2008;64:1383-6.

6. Kerimoglu S, Turgutoglu O, Aynaci O, Turhan AU. Ipsilateral dislocation of the shoulder and elbow joints with contralateral comminuted humeral fracture. Saudi Med J 2006;27:1908-11.

7. Khan MR, Mirdad TM. Ipsilateral dislocation of the shoulder and elbow. Saudi Med J 2001;22:1019-21.

8. Prada C, Romero L, Espinosa Á. Ipsilateral elbow and shoulder dislocation: a case report. J Shoulder Elbow Surg 2019;28:e232-e7.

9. Atik OŞ. Which articles do we prefer to publish? Eklem Hastalik Cerrahisi 2018;29:1.

10. Zacchilli MA, Owens BD. Epidemiology of shoulder dislocations presenting to emergency departments in the United States. J Bone Joint Surg [Am] 2010;92:542-9.

11. Robinson CM, Seah M, Akhtar MA. The epidemiology, risk of recurrence, and functional outcome after an acute traumatic posterior dislocation of the shoulder. J Bone Joint Surg [Am] 2011;93:1605-13.

12. Imerci A, Gölcük Y, Uğur SG, Ursavaş HT, Savran A, Sürer L. Inferior glenohumeral dislocation (luxatio erecta humeri): report of six cases and review of the literature. Ulus Travma Acil Cerrahi Derg 2013;19:41-4.

13. Taylor F, Sims M, Theis JC, Herbison GP. Interventions for treating acute elbow dislocations in adults. Cochrane Database Syst Rev 2012;2012:CD007908.

14. Gottlieb M. Shoulder Dislocations in the Emergency Department: A Comprehensive Review of Reduction Techniques. J Emerg Med 2020;58:647-66.

15. Nho SJ, Dodson CC, Bardzik KF, Brophy RH, Domb BG, MacGillivray JD. The two-step maneuver for closed reduction of inferior glenohumeral dislocation (luxatio erecta to anterior dislocation to reduction). J Orthop Trauma 2006;20:354-7.

16. Groh GI, Wirth MA, Rockwood CA Jr. Results of treatment of luxatio erecta (inferior shoulder dislocation). J Shoulder Elbow Surg 2010;19:423-6.

17. Mallon WJ, Bassett FH 3rd, Goldner RD. Luxatio erecta: the inferior glenohumeral dislocation. J Orthop Trauma 1990;4:19-24.

18. Schliemann B, Heilmann LF, Raschke MJ, Lill H, Katthagen JC, Ellwein A. Isolated fractures of the greater tuberosity: When are they treated conservatively?: A baseline study. Obere Extrem 2018;13:106-11.

19. Koshy JC, Agrawal NA, Seruya M. Nerve Transfer versus Interpositional Nerve Graft Reconstruction for Posttraumatic, Isolated Axillary Nerve Injuries: A Systematic Review. Plast Reconstr Surg 2017;140:953-60.

20. Galvin JW, Eichinger JK. Outcomes Following Closed Axillary Nerve Injury: A Case Report and Review of the Literature. Mil Med 2016;181:e291-7.

21. Palmer SH, Ross AC. Case report. Recovery of shoulder movement in patients with complete axillary nerve palsy. Ann R Coll Surg Engl 1998;80:413-5. 